\title{
An LOD-FDTD Method for the Analysis of Periodic Structures at Normal Incidence
}

\author{
Jun Shibayama, Member, IEEE, Ryoji Ando, Junji Yamauchi, Senior Member, IEEE, and \\ Hisamatsu Nakano, Fellow, IEEE
}

\begin{abstract}
An efficient finite-difference time-domain method based on the locally one-dimensional scheme (LOD-FDTD) is developed for the analysis of periodic structures. The Sherman-Morrison formula is used to efficiently solve the cyclic matrix problem resulting from the application of the periodic boundary condition to the implicit LOD scheme. Through the analysis of a photonic band-gap (PBG) structure, numerical results are found to be identical to those of the alternating-direction implicit (ADI) counterpart. The use of dispersion control parameters enables us to use a large time-step size. As a result, the computational time is reduced to $\simeq 50 \%$ of that of the traditional explicit FDTD while maintaining acceptable numerical results.
\end{abstract}

Index Terms-Alternating-direction implicit (ADI) scheme, finite-difference time-domain (FDTD), periodic structure.

\section{INTRODUCTION}

$\mathbf{F}$ OR the efficient analysis of periodic structures, only a single-unit cell is treated using periodic boundary conditions. One of the frequently used numerical techniques for these structures is the periodic finite-difference time-domain (FDTD) method [1], [2]. Note that as in the conventional FDTD [3], [4], the periodic FDTD suffers from the Courant-Friedich-Levy (CFL) condition with respect to the time-step size $(\Delta t)$. To remove this, Wang et al. [5] have applied the alternating-direction implicit (ADI) scheme [6], [7] to the periodic FDTD, in which the cyclic matrix problem resulting from the implicit scheme is solved using the Sherman-Morrison formula [8]. The periodic ADI-FDTD has also been extended to the analysis of those structures at oblique incidence [9].

As an alternative to the ADI-FDTD, we have proposed the unconditionally stable locally one-dimensional (LOD) FDTD [10], [11]. Nascimento et al. [12], [13] have also independently formulated the LOD-FDTD. The advantage of the LOD-FDTD is that the algorithm is quite simple compared to the ADI-FDTD, maintaining comparable accuracy. While the implicit schemes allow the use of a large $\Delta t$ beyond $\Delta t_{\mathrm{CFL}}$ that is the upper limit determined with the CFL condition, the numerical dispersion error also increases, resulting in accuracy degradation. To reduce the error, Li et al. [14] have introduced the dispersion control parameters [15], [16] into the LOD-FDTD. Note, however,

Manuscript received June 04, 2009; revised June 30, 2009. First published July 28, 2009; current version published August 18, 2009. This work was supported in part by MEXT, Grant-in-Aid for Young Scientists (B) (21760266).

The authors are with the Faculty of Engineering, Hosei University, Tokyo 184-8584, Japan (e-mail: shiba@hosei.ac.jp).

Digital Object Identifier 10.1109/LAWP.2009.2028448 that this improved LOD-FDTD has not been applied to practical problems so far.

The purpose of this letter is to develop an LOD-FDTD for the efficient analysis of periodic structures. To reduce the dispersion error caused by a large $\Delta t$, we introduce the dispersion control parameters [14] to the periodic LOD-FDTD. After presenting the formulation, we analyze a periodic photonic band-gap (PBG) structure [17]. Numerical results are found to be identical to the ADI counterparts, even though the LOD algorithm is simpler than the ADI algorithm. With the control parameters, the numerical results of the periodic LOD-FDTD for $7 \Delta t_{\mathrm{CFL}}$ fairly compare with those of the traditional explicit periodic FDTD. As a result, the computational time is reduced to $\simeq 50 \%$ of that of the explicit FDTD.

\section{FORMULATION}

Maxwell's equations for the two-dimensional (2-D) TE case are expressed as

$$
\frac{\partial \vec{u}}{\partial t}=([A]+[B]) \vec{u}
$$

where $\vec{u}=\left[H_{x}, H_{z}, E_{y}\right]^{T}$ and

$$
[A]=\left[\begin{array}{ccc}
0 & 0 & \frac{\alpha}{\mu} \frac{\partial}{\partial z} \\
0 & 0 & 0 \\
\frac{\alpha}{\epsilon} \frac{\partial}{\partial z} & 0 & 0
\end{array}\right],[B]=\left[\begin{array}{ccc}
0 & 0 & 0 \\
0 & 0 & -\frac{\beta}{\mu} \frac{\partial}{\partial x} \\
0 & -\frac{\beta}{\epsilon} \frac{\partial}{\partial x} & 0
\end{array}\right]
$$

in which $\epsilon$ and $\mu$ represent permittivity and permeability, respectively. $\alpha$ and $\beta$ are the dispersion control parameters in the $z$ and $x$-directions, respectively [14]-[16].

To remove the CFL condition, we apply the Crank-Nicolson scheme to (1) and have

$$
\left([I]+\frac{\Delta t}{2}([A]+[B])\right) \vec{u}^{n+1}=\left([I]-\frac{\Delta t}{2}([A]+[B])\right) \vec{u}^{n}
$$

where $[I]$ denotes the unit matrix. Application of the LOD scheme [10], [12] to (2) gives

$$
\left([I]+\frac{\Delta t}{2}[B]\right) \vec{u}^{*}=\left([I]-\frac{\Delta t}{2}[B]\right) \vec{u}^{n}
$$

for the first step and

$$
\left([I]+\frac{\Delta t}{2}[A]\right) \vec{u}^{n+1}=\left([I]-\frac{\Delta t}{2}[A]\right) \vec{u}^{*}
$$




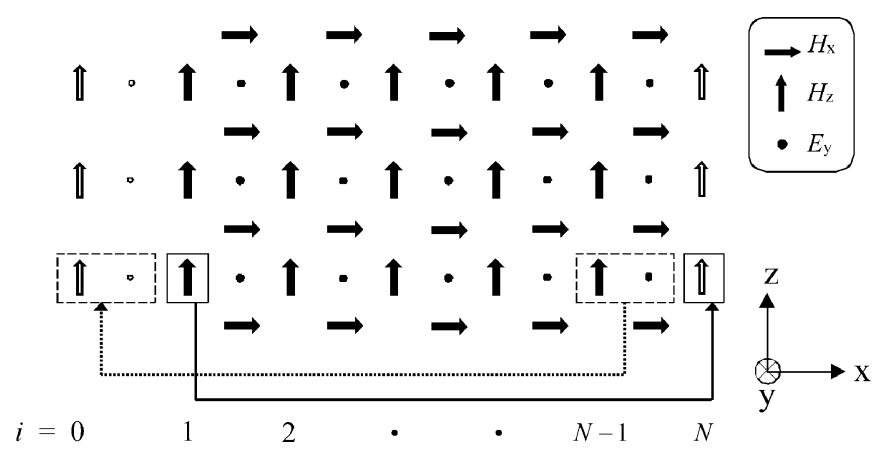

Fig. 1. Field orientation in a unit cell. The periodic boundary condition is imposed when (11) is calculated at $i=1$ and $N-1$.

for the second step, where $\vec{u}^{*}$ is the intermediate field. We finally derive the following basic equations of the improved LODFDTD with the dispersion control parameters [14]:

$$
\begin{aligned}
& H_{x}^{*}=H_{x}^{n} \\
& H_{z}^{*}=H_{z}^{n}-\frac{\beta \Delta t}{2 \mu}\left(\frac{\partial E_{y}^{*}}{\partial x}+\frac{\partial E_{y}^{n}}{\partial x}\right) \\
& E_{y}^{*}=E_{y}^{n}-\frac{\beta \Delta t}{2 \epsilon}\left(\frac{\partial H_{z}^{*}}{\partial x}+\frac{\partial H_{z}^{n}}{\partial x}\right)
\end{aligned}
$$

for the first step and

$$
\begin{aligned}
& H_{x}^{n+1}=H_{x}^{*}+\frac{\alpha \Delta t}{2 \mu}\left(\frac{\partial E_{y}^{n+1}}{\partial z}+\frac{\partial E_{y}^{*}}{\partial z}\right) \\
& H_{z}^{n+1}=H_{z}^{*} \\
& E_{y}^{n+1}=E_{y}^{*}+\frac{\alpha \Delta t}{2 \epsilon}\left(\frac{\partial H_{x}^{n+1}}{\partial z}+\frac{\partial H_{x}^{*}}{\partial z}\right)
\end{aligned}
$$

for the second step. The control parameters $\alpha$ and $\beta$ used in the above formulation contribute to a reduction of the numerical dispersion error. The above equations reduce to those of the conventional LOD-FDTD for $\alpha=\beta=1$. The implementation of the LOD-FDTD is simple due to less arithmetic operations, contributing to the computational time reduction by $10 \%-20 \%$ relative to the ADI-FDTD [10], [11].

Since the infinite periodicity assumed in this letter lies only in the $x$-direction, we impose the periodic boundary condition on the two vertical edges ( $i=1$ and $N-1$ ) of the unit cell as shown in Fig. 1. This requires the implementation of the boundary condition only in the first step where the $x$ derivatives are treated, as discussed below.

In the first step, substituting (7) into (6) gives the following equation to be solved implicitly:

$$
\begin{gathered}
H_{z, i-1, j+1 / 2}^{*}-\left(2+\frac{4 \mu \epsilon \Delta x^{2}}{\beta^{2} \Delta t^{2}}\right) H_{z, i, j+1 / 2}^{*}+H_{z, i+1, j+1 / 2}^{*} \\
=-\frac{4 \mu \epsilon \Delta x^{2}}{\beta^{2} \Delta t^{2}} H_{z, i,+1 / 2}^{n} \\
+\frac{2 \epsilon \Delta x}{\beta \Delta t}\left(E_{y, i+1 / 2, j+1 / 2}^{n}-E_{y, i-1 / 2, j+1 / 2}^{n}+H_{z, i-1, j+1 / 2}^{n}\right. \\
\left.\quad-2 H_{z, i, j+1 / 2}^{n}+H_{z, i+1, j+1 / 2}^{n}\right) .
\end{gathered}
$$

When the edge at $i=1$ is treated, we apply the periodic boundary conditions (see Fig. 1) as

$$
\begin{aligned}
H_{z, 0, j+1 / 2} & =H_{z, N-1, j+1 / 2} \\
E_{y, 1 / 2, j+1 / 2} & =E_{y, N-1 / 2, j+1 / 2}
\end{aligned}
$$

to (11). Similarly, at $i=N-1$, the condition

$$
H_{z, N, j+1 / 2}=H_{z, 1, j+1 / 2}
$$

is used for (11). As a result, we obtain the following matrix:

$$
[M] \vec{x}=\vec{b}
$$

where $\vec{b}$ is the known components of (11), $\vec{x}$ is the unknown components $H_{z}$, and

$$
[M]=\left[\begin{array}{ccccccc}
-c & 1 & 0 & \ldots & \ldots & \ldots & 1 \\
1 & -c & 1 & 0 & \ldots & \ldots & 0 \\
0 & 1 & -c & 1 & 0 & \ldots & 0 \\
\vdots & \ddots & \ddots & \ddots & \ddots & \ldots & \vdots \\
0 & \ldots & 0 & 1 & -c & 1 & 0 \\
0 & \ldots & \ldots & 0 & 1 & -c & 1 \\
1 & \ldots & \ldots & \ldots & 0 & 1 & -c
\end{array}\right]
$$

in which $c=2+4 \mu \epsilon \Delta x^{2} /(\beta \Delta t)^{2}$. Note that $[M]$ is a cyclic matrix not solvable with the Thomas algorithm for a tridiagonal system of linear equations. To efficiently solve (12) with the Thomas algorithm, we take the following approach.

$[M]$ can be rewritten as

$$
[M]=[N]+\overrightarrow{v_{1}}{\overrightarrow{v_{2}}}^{T}
$$

where

$$
[N]=\left[\begin{array}{ccccccc}
-c-1 & 1 & 0 & \ldots & \ldots & \ldots & 0 \\
1 & -c & 1 & 0 & \ldots & \ldots & 0 \\
0 & 1 & -c & 1 & 0 & \ldots & 0 \\
\vdots & \ddots & \ddots & \ddots & \ddots & \ldots & \vdots \\
0 & \ldots & 0 & 1 & -c & 1 & 0 \\
0 & \ldots & \ldots & 0 & 1 & -c & 1 \\
0 & \ldots & \ldots & \ldots & 0 & 1 & -c-1
\end{array}\right]
$$

and $\overrightarrow{v_{1}}=\overrightarrow{v_{2}}=[1,0, \ldots, 0,1]^{T}$.

Here, we resort to the Sherman-Morrison formula [5], [8], [9], leading to

$$
[M]^{-1}=[N]^{-1}-\frac{[N]^{-1}{\overrightarrow{v_{1}}}_{\vec{v}_{2}^{T}}^{T}[N]^{-1}}{1+{\overrightarrow{v_{2}}}^{T}[N]^{-1} \overrightarrow{v_{1}}} .
$$

For the solution of $\vec{x}$ with (14), the following two auxiliary equations are introduced:

$$
\begin{aligned}
& {[N] \overrightarrow{x_{1}}=\vec{b}} \\
& {[N] \overrightarrow{x_{2}}=\overrightarrow{v_{1}} .}
\end{aligned}
$$

Once $\overrightarrow{x_{1}}$ and $\overrightarrow{x_{2}}$ are available, we obtain $\vec{x}$ in (12) as

$$
\vec{x}=\overrightarrow{x_{1}}+A \overrightarrow{x_{2}}
$$




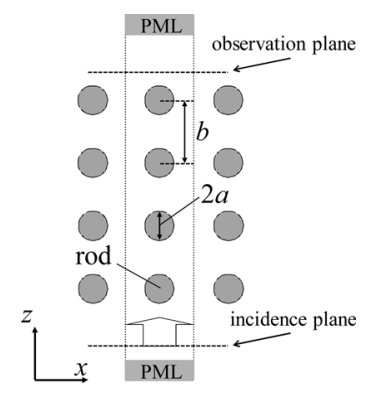

Fig. 2. 2-D PBG structure. Only a single-unit cell is analyzed.

where

$$
A=-\frac{{\overrightarrow{v_{2}}}^{T} \overrightarrow{x_{1}}}{1+{\overrightarrow{v_{2}}}^{T} \overrightarrow{x_{2}}} .
$$

Fortunately, (15) and (16) are tridiagonal systems of linear equations, to which the Thomas algorithm is applicable. Since $\overrightarrow{v_{1}}$ is constant, $\overrightarrow{x_{2}}$ of (16) is solved once and stored throughout the analysis.

The control parameters are derived from the dispersion characteristic equation [14]. Here, we present the parameters with a material refractive index $n\left(\epsilon=\epsilon_{0} n^{2}\right)$ being considered as

$$
\alpha=\frac{n \Delta z}{c \Delta t} \frac{\tan \frac{k c \Delta t}{2}}{\sin \frac{k_{z} n \Delta z}{2}}, \quad \beta=\frac{n \Delta x}{c \Delta t} \frac{\tan \frac{k c \Delta t}{2}}{\sin \frac{k_{x} n \Delta x}{2}}
$$

where $k_{x}=k \cos \phi, k_{z}=k \sin \phi, c$ is the speed of light in a vacuum, and $k(=2 \pi / \lambda)$ is the free-space wavenumber (note that $n$ is not taken into account in [14] since the free space is considered). $\alpha$ and $\beta$ are derived with $\phi=0^{\circ}$ and $90^{\circ}$, respectively, when $n$ is available (for a waveguide problem, $n$ may be chosen to be an effective index of its propagating mode). For sufficiently small $\Delta x$ and $\Delta z, \sin \theta$ is approximated to $\theta$, so the above expressions are simplified to

$$
\alpha=\frac{2 \tan \frac{k c \Delta t}{2}}{c \Delta t k_{z}}, \beta=\frac{2 \tan \frac{k c \Delta t}{2}}{c \Delta t k_{x}} .
$$

Notice that knowledge regarding $n$ is not required in (19). In other words, the control parameters can be used without paying attention to the background index, provided that spatial sampling widths are sufficiently small. In Section III, we will examine numerical results with both (18) and (19).

\section{NUMERICAL RESULTS}

To investigate the periodic LOD-FDTD in comparison with the explicit periodic FDTD, we analyze a 2-D PBG structure treated in [17] at normal incidence. Fig. 2 shows the PBG structure to be analyzed, in which the radius of the dielectric rod is $a=2 \mathrm{~mm}$ and its dielectric constant is $\epsilon_{r}=4.2$ (surrounded by air). The rods are arranged in a square lattice such that the center-to-center separation distance $b=9 \mathrm{~mm}$ is equal to the unit cell width. A Gaussian pulse plane wave is launched from the incidence plane (see Fig. 2). The frequency characteristics of the transmission are calculated using the time-domain response in the observation plane. The rod is discretized by 16 cells across

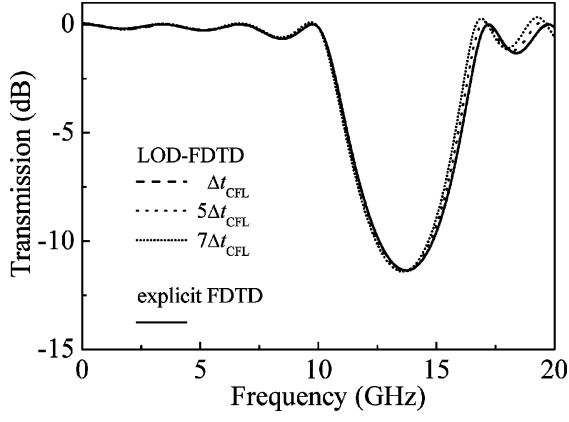

(a) LOD-FDTD

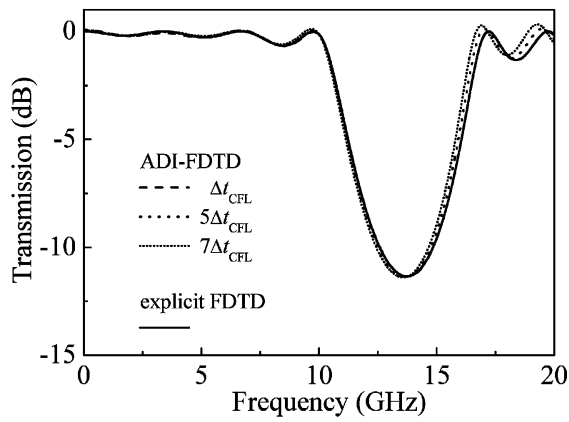

(b) ADI-FDTD

Fig. 3. Transmission versus frequency. (a) LOD-FDTD and (b) ADI-FDTD. The dispersion control parameters are $\alpha=\beta=1$ (conventional LOD- and ADI-FDTDs). LOD and ADI results for $\Delta t_{\mathrm{CFL}}$ are almost superimposed on the result of the explicit FDTD.

its diameter, leading to $\Delta x=\Delta z=0.25 \mathrm{~mm}$. The number of sampling points is $N_{x} \times N_{z}=38 \times 220$, including 20-cell perfectly matched layer (PML) regions [18] for both input and output ports.

Fig. 3(a) shows the transmission obtained from the LODFDTD with $\alpha=\beta=1$. For reference, the transmission from the explicit FDTD is also included with $\Delta t_{\mathrm{CFL}}=0.575 \mathrm{~ns}$ (which is slightly smaller than the actual upper limit for stability). It is seen that the transmission of the LOD-FDTD with a large $\Delta t$ slightly deviates from that of the explicit FDTD in the high-frequency region, which stems from the numerical dispersion error. It is noteworthy that the LOD results are identical to the ADI results shown in Fig. 3(b), even though the former is first-order accurate in time while the latter is second-order accurate. The noncommutativity error of the LOD-FDTD is negligible for the problem treated here.

To achieve a more accurate result, we employ the improved LOD-FDTD with the dispersion control parameters being selected. For example, we obtain $\alpha=\beta=1.01255$ from (18) with $\Delta x=\Delta z=0.25 \mathrm{~mm}, \Delta t=7 \Delta t_{\mathrm{CFL}}, \lambda$ at $15 \mathrm{GHz}$, and $n$ derived from the ratio between the rod and air areas in a single cell. On the other hand, (19) yields $\alpha=\beta=1.01215$, which are almost the same as the above. Fortunately, the inclusion of the control parameters imposes no computational burden [14]. Fig. 4 depicts the transmission with (18). The results are found to be improved, in which the transmission for $5 \Delta t_{\mathrm{CFL}}$ is almost superimposed on the explicit counterpart. In addition, the result for $7 \Delta t_{\mathrm{CFL}}$ is acceptable, whereas only a slight deviation is seen in the high-frequency region. Interestingly, the use 


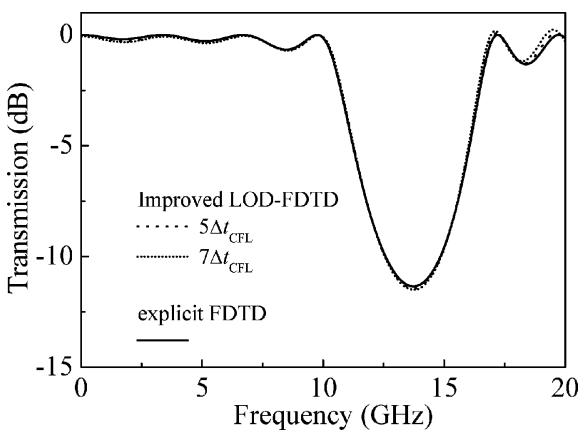

Fig. 4. Transmission obtained from the improved LOD-FDTD with the dispersion control parameters.

of (19) reproduces almost the same numerical results as those in Fig. 4, although not explicitly shown. This means that no knowledge regarding $n$ is required, provided that the spatial sampling widths are reasonably small.

Finally, we mention the computational efficiency of the periodic LOD-FDTD. Note that the relative efficiency to the conventional FDTD depends on the computer used and the computational code developed. In our Fortran code, the computational time of the LOD-FDTD for $\simeq 4 \Delta t_{\mathrm{CFL}}$ is comparable to that of the explicit FDTD when a PC with a Core(TM)2 Quad processor $(3.0 \mathrm{GHz})$ is used. The computational time is reduced by $\simeq 25 \%$ and $50 \%$ for $5 \Delta t_{\mathrm{CFL}}$ and $7 \Delta t_{\mathrm{CFL}}$, respectively, compared to the explicit counterpart, demonstrating an efficiency improvement of the LOD-FDTD.

\section{CONCLUSION}

An efficient numerical technique for periodic structures has been developed on the basis of the LOD-FDTD method. Analysis of a PBG structure reveals that the results of the periodic LOD-FDTD are identical to the ADI counterparts. The use of the dispersion control parameters contributes to an accuracy improvement particularly in the high-frequency region, in which no knowledge of the material refractive index is required. With acceptable results, the computational time for $7 \Delta t_{\mathrm{CFL}}$ is reduced to $\simeq 50 \%$ of that of the explicit FDTD. Extension to periodic structures at oblique incidence [9], [17] is left for a future study.

\section{REFERENCES}

[1] W.-J. Tsay and D. M. Pozar, "Application of the FDTD technique to periodic problems inscattering and radiation," IEEE Microw. Guided Wave Lett., vol. 3, no. 8, pp. 250-252, Aug. 1993.

[2] P. Harms, R. Mittra, and W. Ko, "Implementation of the periodic boundary condition in the finite-difference time-domain algorithm for FSS structures," IEEE Trans. Antennas Propag., vol. 42, no. 9, pp. 1317-1324, Sep. 1994

[3] A. Taflove and S. C. Hagness, Computational Electrodynamics: The Finite-Difference Time-Domain Method, 3rd ed. Norwood, MA: Artech House, 2005.

[4] T. Uno, Finite Difference Time Domain Method for Electromagnetic Field and Antenna Analyses. Tokyo, Japan: Corona, 1998.

[5] S. Wang, J. Chen, and P. Ruchhoeft, "An ADI-FDTD method for periodic structuers," IEEE Trans. Antennas Propag., vol. 53, no. 7, pp. 2343-2346, Jul. 2005.

[6] T. Namiki, "A new FDTD algorithm based on alternating-direction implicit method," IEEE Trans. Microw. Theory Tech., vol. 47, no. 10, pp. 2003-2007, Oct. 1999.

[7] F. Zheng, Z. Chen, and J. Zhang, "Toward the development of a three-dimensional unconditionally stable finite-difference time-domain method," IEEE Trans. Microw. Theory Tech., vol. 48, no. 9, pp. $1550-1558$, Sep. 2000

[8] J. W. Thomas, Numerical Partial Differential Equations: Finite Difference Methods. Berlin, Germany: Springer-Verlag, 1995.

[9] Y. Mao, B. Chen, H. Chen, and Q. Wu, "Unconditionally stable SFDTD algorithm for solving oblique incident wave on periodic structures," IEEE Microw. Wireless Compon. Lett., vol. 19, no. 5, pp. 257-259, May 2009

[10] J. Shibayama, M. Muraki, J. Yamauchi, and H. Nakano, "Efficient implicit FDTD algorithm based on locally one-dimensional scheme," Electron. Lett., vol. 41, no. 19, pp. 1046-1047, Sep. 2005.

[11] J. Shibayama, M. Muraki, R. Takahashi, J. Yamauchi, and H. Nakano, "Performance evaluation of several implicit FDTD methods for optical waveguide analyses," J. Lightw. Technol., vol. 24, no. 6, pp. 2465-2472, Jun. 2006

[12] V. E. do Nascimento, J. A. Cuminato, F. L. Teixeira, and B.-H. V. Borges, "Unconditionally stable finite-difference time-domain method based on the locally-one-dimensional technique," in Proc. 22nd Symp. Brasileiro Telecomun., Campinas, Brazil, Sep. 2005, pp. 288-291.

[13] V. E. do Nascimento, B.-H. V. Borges, and F. L. Teixeira, "Split-field PML implementations for the unconditionally stable LOD-FDTD method," IEEE Microw. Wireless Compon. Lett., vol. 16, no. 7, pp. 398-400, Jul. 2006

[14] E. Li, I. Ahmed, and R. Vahldieck, "Numerical dispersion analysis with an improved LOD-FDTD method," IEEE Microw. Wireless Compon. Lett., vol. 17, no. 5, pp. 319-321, May 2007.

[15] S. Wang and F. L. Teixeira, "Dispersion-relation-preserving FDTD algorithms for large-scale three-dimensional problems," IEEE Trans. Antennas Propag., vol. 51, no. 8, pp. 1818-1828, Aug. 2003.

[16] T. T. Zygiridis and T. D. Tsiboukis, "Development of higher order FDTD schemes with controllable dispersion error," IEEE Trans. Antennas Propag., vol. 53, no. 9, pp. 2952-2960, Sep. 2005.

[17] J. A. Roden, S. D. Gedney, M. P. Kesler, J. G. Maloney, and P. H. Harms, "Time-domain analysis of periodic structures at oblique incidence: Orthogonal and nonorthogonal FDTD implementations," IEEE Trans. Microw. Theory Tech., vol. 46, no. 4, pp. 420-427, Apr. 1998.

[18] J. P. Berenger, "Numerical reflection from FDTD-PMLs: A comparison of the split PML with the unsplit and CFS PMLs," IEEE Trans. Antennas Propag., vol. 50, no. 3, pp. 258-265, Mar. 2002. 